\title{
Communication
}

\section{Preparation of Porous Liquid Based on Silicalite-1}

\author{
Yutong Liu ${ }^{1,2}$, Yang Bai ${ }^{1}$ and Tao Tian ${ }^{1, *(D)}$ \\ 1 Key Laboratory of Groundwater Resources and Environment, Ministry of Education, College of \\ Environment and Resource, Jilin University, Changchun 130012, China; liuyt841011@163.com (Y.L.); \\ 15943013272@163.com (Y.B.) \\ 2 Jilin Engineering Normal University, Changchun 130012, China \\ * Correspondence: tiantao@jlu.edu.cn
}

Received: 25 October 2019; Accepted: 29 November 2019; Published: 1 December 2019

\begin{abstract}
Solid porous materials, like zeolites, have been widely used in a variety of fields such as size-and-shape-selective absorption/separation and catalysis because of their porosity. However, there are few liquid materials that exhibit permanent porosity. Porous liquids are a novel material that combine the properties of fluidity and permanent porosity. They have potential applications in many fields such as gas separation, storage and transport. Herein, we report a novel Type 1 porous liquid prepared based on silicalite-1. The pore size of this porous liquid was determined by positron annihilation lifetime spectroscopy (PALS), and the $\mathrm{CO}_{2}$ capacities were determined by the intelligent gravimetric analyzer (IGA). The unique properties of this porous liquid can promote its application in many fields such as gas storage and transport.
\end{abstract}

Keywords: porous liquid; silicalite- $1 ; \mathrm{CO}_{2}$ capacities; gas storage

\section{Introduction}

Zeolites, a family of microporous aluminosilicates characterized by a regular system of inner channels and uniform open pores [1,2], have been widely used in size-and-shape-selective absorption/separation [3-11] and catalysis [12-15] due to their sharp pore size distribution, large surface area and thermal/hydrothermal stability. When zeolites are used as adsorbents, they have high adsorption capacity and selectivity for a variety of gases such as $\mathrm{He}$ [4], $\mathrm{CO}_{2}$ [7], $\mathrm{O}_{2}$ [10], $\mathrm{N}_{2}, \mathrm{CH}_{4}$, $\mathrm{C}_{2} \mathrm{H}_{6}[11]$ and so on.

As solid adsorbents, they have many advantages, such as lower energy penalties in adsorption-desorption cycles, but they are difficult to apply in conventional liquid processes because of their solid properties $[16,17]$. One way to solve this problem is by liquefiying the zeolites into porous liquids that combine the properties of fluidity and permanent porosity.

There are three types of porous liquids proposed by James et al. based on the nature of the host systems [16]. Type 1 is neat liquid hosts that cannot collapse or interpenetrate; type 2 and 3 are rigid hosts or particles of microporous frameworks dispersed in sterically hindered solvents, respectively. By now, several porous liquids have been reported. Dai's group had reported a Type 1 porous liquid using hollow silica spheres as hosts (denoted by OS@HS) and determined the gas separation for $\mathrm{N}_{2} / \mathrm{CO}_{2}$ [18]. Although the gas permeability and selectivity are moderately low, the good tunability of this hybrid system affords ample choices for further optimization. James and co-workers had prepared "porous liquids" by dissolving rigid organic cage molecules into a sterically hindered solvent; the solubility of methane gas in this liquid increased about 8 times [17]. We have reported a class of Type 3 porous liquids synthesized by solution mixing of porous hosts (ZIF-8, ZSM-5 and Silicalite-1) and rationally designed ionic liquids [19]. The $\mathrm{CO}_{2}$ capacity measurements confirm the permanent porosity in these liquids. These liquids have potential applications in many fields such as gas separation, storage and transport. 
Herein, a Type 1 porous liquid based on nanosized silicalite-1 (S-1) zeolite, denoted as S-1-Liquid, has been successfully prepared. The compound was characterized by Fourier-transform infrared (FT-IR), thermogravimetric analysis (TGA), X-ray diffraction (XRD), $\mathrm{N}_{2}$ adsorption/desorption, scanning electron microscopy (SEM) and transmission electron microscopy (TEM). The positron annihilation lifetime spectroscopy (PALS) and $\mathrm{CO}_{2}$ capacities determined by the intelligent gravimetric analyzer (IGA) were used to demonstrate its porosity.

\section{Materials and Methods}

The Type 1 porous liquid (S-1-Liquid) was prepared in a three-step synthetic procedure, as shown in Scheme 1.

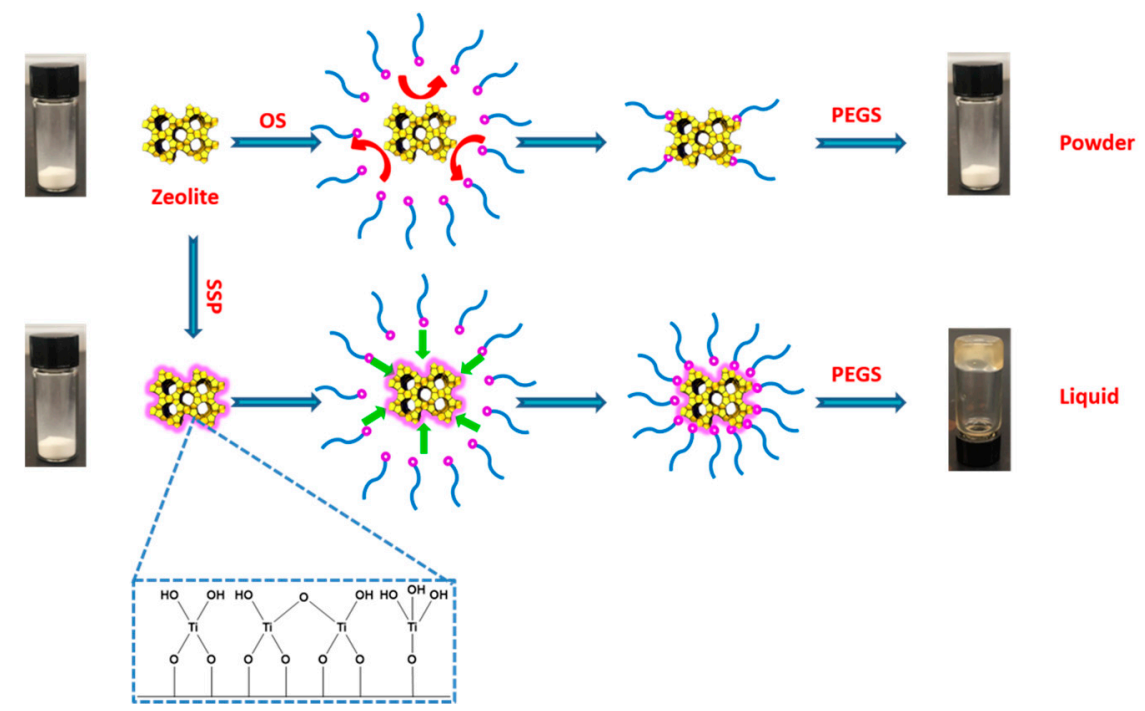

Scheme 1. Preparation of porous liquid with nanosized silicalite-1 zeolite. (a) Scheme without SSP; (b) Scheme with SSP. OS = organosilane; PEGS = poly(ethylene glycol) tailed sulfonate; SSP = surface sol-gel process.

\subsection{Synthesis of Nanosized Silicalite-1}

Nanosized S-1 zeolite has been synthesized under direct hydrothermal conditions [20]. The initial solutions were prepared by mixing tetraethyl orthosilicate (98\%, Aldrich, Shanghai, China) and tetrapropylammonium hydroxide (1 M aqueous solution, Aldrich, Shanghai, China). The chemical composition is 9 TPAOH: $25 \mathrm{SiO}_{2}: 480 \mathrm{H}_{2} \mathrm{O}$. The silica alkoxides were hydrolyzed under slow stirring $(50 \mathrm{rpm})$ for $24 \mathrm{~h}$ at room temperature. The obtained clear solutions were transferred in polypropylene bottles and subjected to hydrothermal treatment in a conventional oven. The syntheses were performed at $90{ }^{\circ} \mathrm{C}$ for $96 \mathrm{~h}$. After the treatment, the zeolite suspensions were purified in a series of four steps consisting of high-speed centrifugation $(10,000 \mathrm{rpm}, 20 \mathrm{~min})$, removal of the mother liquor, and washed by water for several times. After that, the sample was dried at $80{ }^{\circ} \mathrm{C}$ in the oven overnight and calcined at $600{ }^{\circ} \mathrm{C}$ for $6 \mathrm{~h}$ to remove organic impurity.

\subsection{Surface Sol-gel Process (SSP) on Silicalite-1}

The SSP on S-1 surface was based on literature reports [21]. Typically, $1.0 \mathrm{~g}$ of pre-dried S-1 zeolite powder was added into a reactor under an anhydrous condition. Subsequently, $5 \mathrm{~mL}$ of titanium(IV) butoxide (97\%, Aldrich, Shanghai, China) in a mixture of $10 \mathrm{~mL}$ of anhydrous toluene (99.8\%, Aldrich, Shanghai, China) and $10 \mathrm{~mL}$ of anhydrous methanol (99.8\%, Aldrich, Shanghai, China) was added into the reactor through a syringe. After $30 \mathrm{~min}$, the precipitate was separated and washed with anhydrous methanol to remove the unreacted titanium(IV) butoxide. After that, an excess amount of water was 
mixed with the resulting sample to hydrolyze the monolayer of titanium oxide. Finally, the sample was washed with anhydrous methanol several times and dried at $40^{\circ} \mathrm{C}$ in a vacuum oven.

\subsection{Preparation of S-1-Liquid}

A total of $1.0 \mathrm{~g}$ of S-1 was dispersed in $20 \mathrm{~mL}$ deionized water and sonicated for $10 \mathrm{~min}$. Then, $2.0 \mathrm{~mL}$ of organosilane (OS), $\left(\mathrm{CH}_{3} \mathrm{O}\right)_{3} \mathrm{Si}\left(\mathrm{CH}_{2}\right)_{3} \mathrm{~N}^{+}\left(\mathrm{CH}_{3}\right)\left(\mathrm{C}_{10} \mathrm{H}_{21}\right)_{2} \mathrm{Cl}$ (Aldrich, Shanghai, China), was added into the suspension under vigorous stirring. Subsequently, the mixture was aged at room temperature for $24 \mathrm{~h}$. After that, the precipitate, denoted as OS@S-1, was washed with water for three times and with ethanol for three times, respectively, and dried at $100{ }^{\circ} \mathrm{C}$ overnight. Finally, the OS@S-1 was treated by $15.0 \mathrm{~mL}$ poly(ethylene glycol) tailed sulfonate (PEGS, 16.5\%, $\mathrm{C}_{9} \mathrm{H}_{19}-\mathrm{C}_{6} \mathrm{H}_{4}-\left(\mathrm{OCH}_{2} \mathrm{CH}_{2}\right)_{20} \mathrm{O}-\left(\mathrm{CH}_{2}\right)_{3} \mathrm{SO}_{3} \mathrm{~K}^{+}$, Aldrich, Shanghai, China) at $70{ }^{\circ} \mathrm{C}$ for $24 \mathrm{~h}$. The mixture was dried at $70{ }^{\circ} \mathrm{C}$ and dispersed in $15.0 \mathrm{~mL}$ acetone for three times to remove the $\mathrm{KCl}$ byproduct. The sample was dried at $70{ }^{\circ} \mathrm{C}$ for $24 \mathrm{~h}$ to obtain the S-1-Liquid. The S-1-Liquid was kept at $40{ }^{\circ} \mathrm{C}$ under vacuum for use.

In the beginning, nanosized S-1 zeolite was used directly to prepare the S-1-Liquid, as shown in Scheme 1a. However, disappointingly, after the replace of chloride anion of the OS by PEGS, we cannot obtain a stable liquid phase. It is probably because there are too few hydroxy groups on the surface of the S-1 zeolite. Then, we changed the strategy. The surface sol-gel process (SSP) was used to increase the surface hydroxy groups of S-1 zeolite before the surface modification by OS, as shown in Scheme $1 \mathrm{~b}$, yielding an optically transparent zeolite-based porous liquid (S-1-Liquid) at room temperature.

\section{Results and Discussion}

The Fourier-transform infrared (FT-IR) spectra of products in each step are presented in Figure 1a. For the nanosized S-1 zeolite, the absorption peak at $799 \mathrm{~cm}^{-1}$ is due to the symmetric stretching vibrations of the Si-O-Si bonds of zeolite and the bands at $1074 \mathrm{~cm}^{-1}$ and $1225 \mathrm{~cm}^{-1}$ are attributed to the asymmetric stretching vibrations of the Si-O-Si bonds. The IR spectrum of S-1 after SSP is closely related to that of S-1 (Figure S1). After surface modification, some featured bands assigned to the OS moiety are clearly visible in the OS@S-1 spectrum, such as the stretching and bending vibrations of $-\mathrm{CH}_{2}$ - backbones at $2928 \mathrm{~cm}^{-1}, 2870 \mathrm{~cm}^{-1}$ and $1470 \mathrm{~cm}^{-1}$. The bands at $799 \mathrm{~cm}^{-1}, 1074 \mathrm{~cm}^{-1}$ and $1225 \mathrm{~cm}^{-1}$, which are attributed to the stretching vibrations of the Si-O-Si bonds of zeolite, are further enhanced. In the spectrum of the S-1-Liquid, additional bands such as $2868 \mathrm{~cm}^{-1}, 1724 \mathrm{~cm}^{-1}, 1186$ $\mathrm{cm}^{-1}$ and $1094 \mathrm{~cm}^{-1}$ are attributed to the aliphatic, phenyl, sulfonate and ether, respectively. It means that the OS@S-1 is surrounded by the PEGS completely to obtain the S-1-Liquid. The S-1 after SSP was examined by XPS to determine the existence of titanium species on the surface of S-1. Figure S2 shows the XPS spectrum on the $\mathrm{P}_{2} \mathrm{p}$ region of the S-1 after SSP; two emission peaks are observed at $458.0 \mathrm{eV}$ and $464.0 \mathrm{eV}$ for $\mathrm{Ti} 2 \mathrm{p}_{3 / 2}$ and $\mathrm{Ti} 2 \mathrm{p}_{1 / 2}$, respectively, indicating the existence of titanium in the $\mathrm{Ti}^{4+}$ state on the surface of S-1.


Figure 1. FT-IR spectra (a) and TGA trace (b) of S-1, OS@S-1 and S-1-Liquid. 
Figure $1 \mathrm{~b}$ shows the thermogravimetric analysis (TGA) of S-1, OS@S-1, and S-1-Liquid. There is about $2.7 \%$ of mass loss during heating until the temperature reaches $700{ }^{\circ} \mathrm{C}$ for the $\mathrm{S}-1$, according to the removal of the adsorption water. The S- 1 after treated by SSP has a mass loss of about $2.8 \%$ (Figure S2), similar to the S-1, which means that there are no organic group residue on S-1 surface after SSP. For the OS@S-1, the loss weight is about 13.3\%, corresponding to the decomposition of OS. In comparison, when the S-1 before treated by SSP reacts with the OS, the mass loss is about $6.8 \%$, much lower than OS@S-1 (Figure S3). This demonstrates that the S-1 after SSP has more surface hydroxyl compared to the S-1 to react with the OS. It means that the surface sol-gel process is efficient to increase the surface hydroxyl of S-1. There is no significant mass loss during heating until the temperature reaches approximately $190^{\circ} \mathrm{C}$, demonstrating that the S-1-Liquid is solvent-free. From $190^{\circ} \mathrm{C}$ to $460^{\circ} \mathrm{C}$, there is $67 \%$ of mass loss for S-1-Liquid, corresponding to the decomposition of organic groups around the S-1 surfaces. The final loss weight is $66.5 \%$ when the temperature approaches $700{ }^{\circ} \mathrm{C}$.

As shown in Figure 2a, the X-ray diffraction pattern of S-1 shows a typical characteristic diffraction pattern of the MFI-type zeolite. The four peaks at $2 \theta$ of $8.08,9.04,23.3$ and $24.1^{\circ}$ corresponded to the (101), (200), (501) and (303) planes respectively [3]. The S-1-Liquid has a similar XRD pattern with S-1, indicating the structure of S-1 has been preserved in the liquid. To confirm that the pores of S-1 zeolite remained empty after OS modification, OS@S-1 was also characterized by $\mathrm{N}_{2}$ adsorption/desorption isotherms (Figure $2 \mathrm{~b}$ ). There was no significant change in the $\mathrm{N}_{2}$ adsorption/desorption isotherm for OS@S-1, compared to S-1 zeolite. It means that the pores of S-1 zeolite remained empty after surface modification by OS. This is an important result to demonstrate that the OS@S-1 still has empty pores for gas transport or storage.
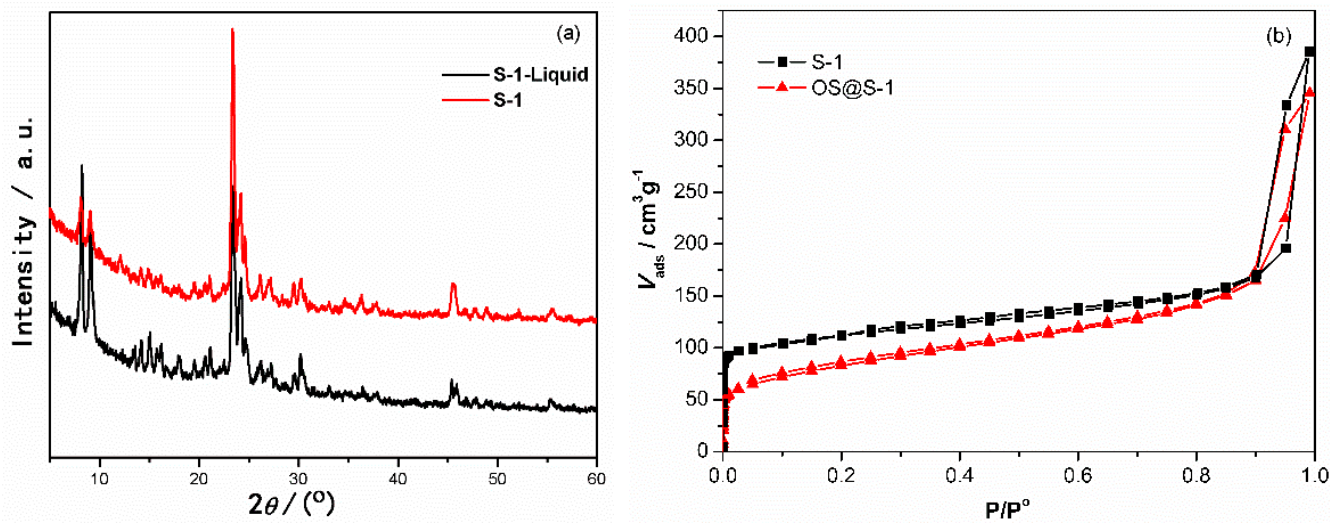

Figure 2. XRD patterns of S-1 and S-1-Liquid (a) and $\mathrm{N}_{2}$ adsorption/desorption isotherms of S-1 and OS@S-1 samples (b).

The as-synthesized nanosized S-1 and OS@S-1 were determined by transmission electron microscopy (TEM), as shown in Figure S4. The crystal size of S-1 is around $100 \mathrm{~nm}$, and the size distribution of OS@S-1 is about $150 \mathrm{~nm}$ to $200 \mathrm{~nm}$, which is larger than that of S-1 because of the surface modification by OS. The nanostructure of S-1-Liquid was imaged by scanning electron microscopy (SEM) and (TEM). As observed in Figure 3, the S-1 nanocrystals are defined well in the PEGS to form a liquid-like polymeric medium. These nanocrystals are combined together to form large agglomerates through the strong interaction among the surface functional groups. 


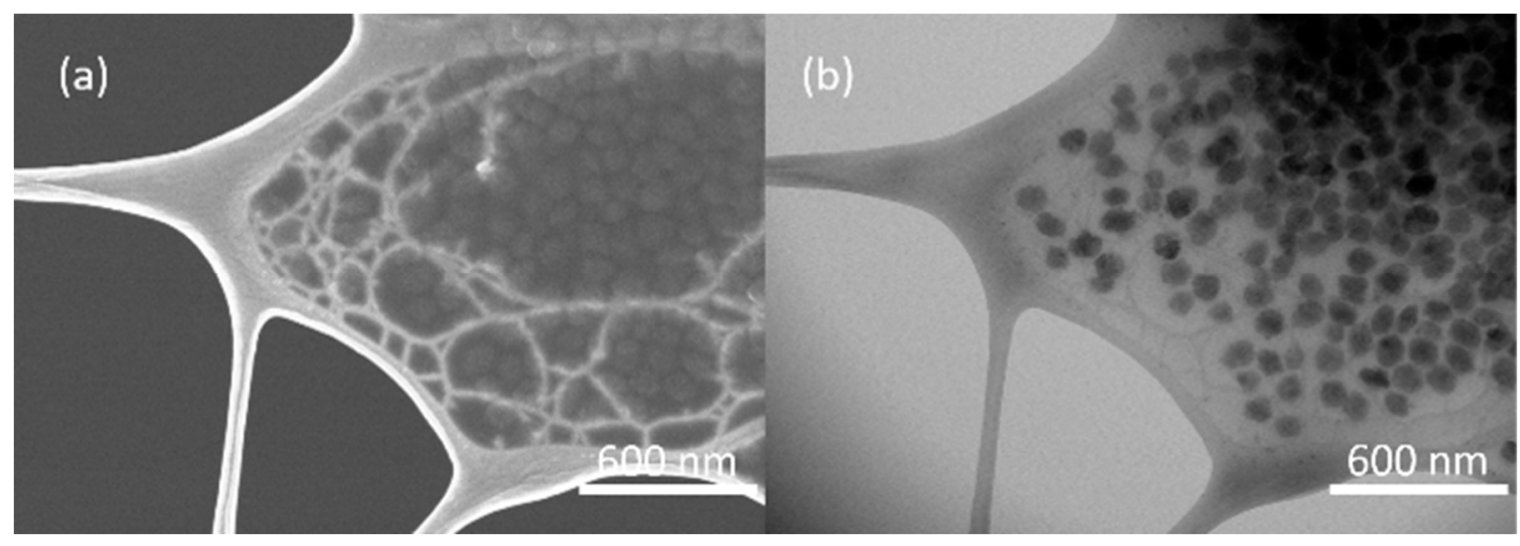

Figure 3. SEM (a) and TEM images (b) of S-1-Liquid.

Positron annihilation lifetime spectroscopy (PALS) was then used to determine the average size of the pores contained within the studied S-1-Liquid. The PALS technique measures the lifetime and intensity of positions that annihilate in materials when the material of interest is exposed to a positron source, which is ${ }^{22} \mathrm{NaCl}$ in this study. Once entering the materials, positrons experience thermalization, diffusion, and finally are trapped and annihilated by electrons. The positron lifetime is dependent on the overlap of the positron wavefunction with the wavefunctions of the electrons in materials. Therefore, the electron density distribution in materials, in particular, the presence of open volume, can be obtained by using this technique. The positron lifetime is defined as the time interval between the injection of positions into the materials (indicated by a birth gamma ray of $1.274 \mathrm{MeV}$ ) and the decay of the positron-electron pairs (indicated by two $0.511 \mathrm{MeV}$ gamma rays traveling in opposite directions). Data were collected using a digital oscilloscope with a system timing resolution of $173 \mathrm{ps.}$ More details about the PALS system could be found in Reference [22].

Figure 4 shows the recorded positron lifetime spectrum as well as the fitting curve by applying 3-component analysis. The first lifetime $(\tau 1)$ indicates the positrons being annihilated in the bulk of the materials ( $<200 \mathrm{ps})$. The second lifetime, $\tau 2$, is attributed to the positrons being annihilated in defects (300-500 ps). The third lifetime, $\tau 3$, refers to ortho-positronium (o-Ps), a parallel spin complex of a positron and electron, which forms in low electron density regions, such as free volumes, holes, interfaces, and pores. The o-Ps lifetime and intensity are often associated with the size and concentration, respectively, of the open volume in materials. From the fitting procedure one obtains positron lifetimes and intensities. The o-Ps lifetime, $\tau 3$, is typically related to the average radius of a free volume element, $r$, which is assumed to be spherical, by Tao-Eldrup model,

$$
\tau_{3}=\frac{1}{2}\left(1-\frac{r}{r+\Delta r}+\frac{1}{2 \pi} \sin \left[2 \pi \frac{r}{r+\Delta r}\right]\right)^{-1}
$$

where $\Delta \mathrm{r}$ is the empirical electron layer thickness, which is taken to be $1.66 \mathrm{~A}[23,24]$. 


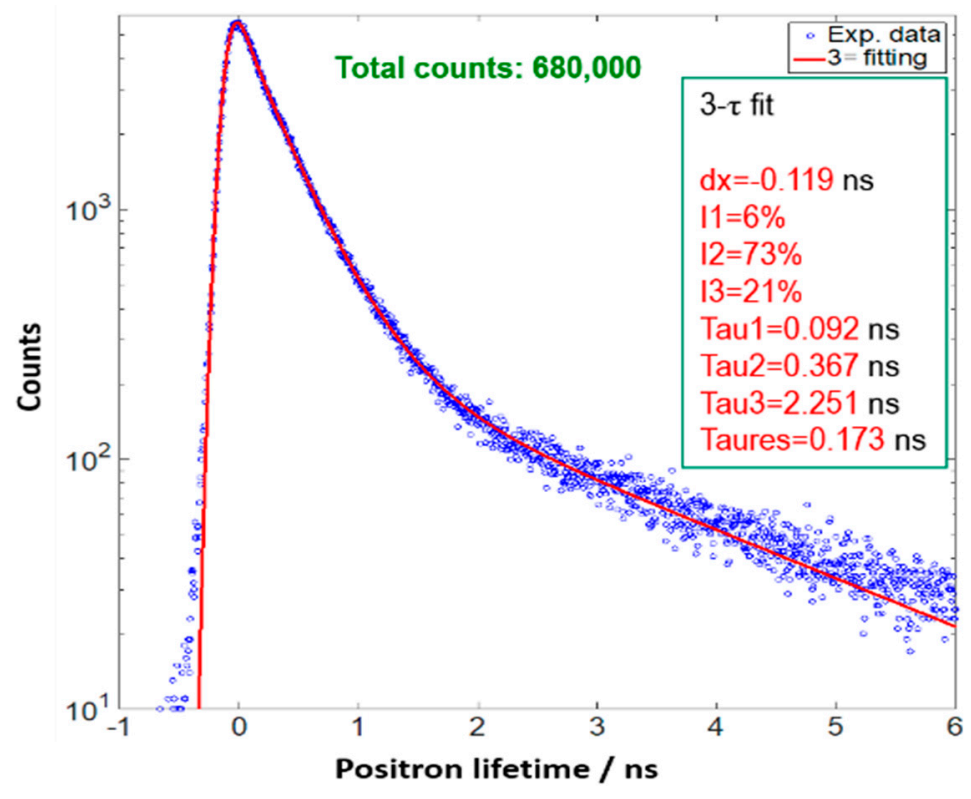

Figure 4. The positron lifetime traces of S-1-Liquid collected at $298 \mathrm{~K}$.

By applying Equation (1), the o-Ps lifetime of 2.251 ns correlates to an average pore size of 0.61 $\mathrm{nm}$ in the S-1-Liquid; this is consistent with the pore size of S-1 zeolite.

To further prove that the pores of S-1 are still empty in the S-1-Liquid, the $\mathrm{CO}_{2}$ capacities of it were determined by an Intelligent Gravimetric Analyzer. The $\mathrm{CO}_{2}$ uptake capacity of S-1-Liquid is enhanced compared to the PEGS, as shown in Figure 5. The $\mathrm{CO}_{2}$ uptake value of S-1-Liquid is $0.474 \mathrm{wt} \%$ at the pressure of 1 bar, while the uptake value of PEGS is $0.261 \mathrm{wt} \%$. When the pressure increases to $10 \mathrm{bar}$, the $\mathrm{CO}_{2}$ uptake value of S-1-Liquid is $2.524 \mathrm{wt} \%$, which remains higher than that of PEGS $(2.261 \mathrm{wt} \%)$. Both the S-1-Liquid and PEGS have hysteresis loops. The S-1-Liquid remains more $\mathrm{CO}_{2}$ as the pressure decreases during the desorption process due to the permanent porosity. The $\mathrm{CO}_{2}$ uptake value of S-1-Liquid is $1.406 \mathrm{wt} \%$ at $1 \mathrm{bar}$, much higher than that of PEGS ( $0.809 \mathrm{wt} \%)$. There is $55.7 \% \mathrm{wt} \%$ of $\mathrm{CO}_{2}$ remains in the S-1-Liquid, demonstrating the potential application for $\mathrm{CO}_{2}$ storage.

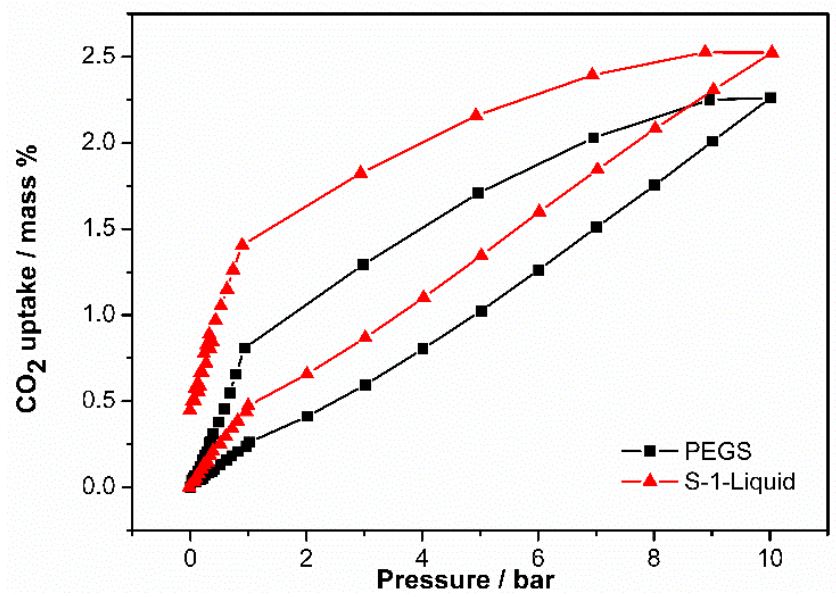

Figure 5. $\mathrm{CO}_{2}$ adsorption-desorption isotherms of S-1-Liquid and PEGS collected below 10 bar at $298 \mathrm{~K}$.

\section{Conclusions}

In summary, a Type 1 porous liquid S-1-Liquid was synthesized in a three-step synthetic procedure. This porous liquid is stable and homogeneous at room temperature. It exhibits enhanced $\mathrm{CO}_{2}$ adsorption capacity due to the permanent porosity, which was also confirmed by PALS results. We believe that this porous liquid has potential applications in many fields such as gas storage and transport. 
Supplementary Materials: The following are available online at http://www.mdpi.com/1996-1944/12/23/3984/s1, Figure S1: FT-IR spectra of S-1 and S-1 treated by SSP; Figure S2: XPS spectra of the $\mathrm{P}_{2} \mathrm{p}$ region of S-1 treated by SSP.; Figure S3: TGA trace of S-1, S-1 after SSP, OS@S-1 and OS@S-1 after SSP. Figure S4: TEM images of S-1 (a, b) and OS@S-1 (c, d).

Author Contributions: Conceptualization, T.T.; methodology, T.T.; investigation, Y.L.; resources, T.T.; data curation, Y.L.; writing — original draft preparation, Y.L.; writing—review and editing, T.T.; visualization, Y.B.; funding acquisition, T.T.

Funding: This work was funded by National Natural Science Foundation of China (21771080), Science and Technology Project of the "13th Five-Year Plan" of Jilin Provincial Department of Education (JJKH20180166KJ).

Acknowledgments: We are thankful for support of this work by the State Key Laboratory of Inorganic Synthesis and Preparative Chemistry, Jilin University.

Conflicts of Interest: The authors declare no conflict of interest.

\section{References}

1. Corma, A. Inorganic Solid Acids and Their Use in Acid-Catalyzed Hydrocarbon Reactions. Chem. Rev. 1995, 95, 559-614. [CrossRef]

2. Yu, J.H.; Xu, R.R. Insight into the construction of open-framework aluminophosphates. Chem. Soc. Rev. 2006, 35, 593-604. [CrossRef] [PubMed]

3. Xu, C.C.; Lu, X.F.; Wang, Z.B. Effects of sodium ions on the separation performance of pure-silica MFI zeolite membranes. J. Membr. Sci. 2017, 524, 124-131. [CrossRef]

4. Ye, P.C.; Grahn, M.; Korelskiy, D.; Hedlund, J. Efficient Separation of $\mathrm{N}_{2}$ and He at Low Temperature Using MFI Membranes. AIChE J. 2016, 62, 2833-2842. [CrossRef]

5. Zhang, H.; Xiao, Q.; Guo, X.; Li, N.; Kumar, P.; Rangnekar, N.; Jeon, M.Y.; Al-Thabaiti, S.; Narasimharao, K.; Basahel, S.N.; et al. Open-Pore Two-Dimensional MFI Zeolite Nanosheets for the Fabrication of Hydrocarbon-Isomer-Selective Membranes on Porous Polymer Supports. Angew. Chem. Int. Ed. 2016, 55, 7184-7187. [CrossRef]

6. Xia, S.X.; Peng, Y.; Wang, Z.B. Microstructure manipulation of MFI-type zeolite membranes on hollow fibers for ethanol-water separation. J. Membr. Sci. 2016, 498, 324-335. [CrossRef]

7. Sjöberg, E.; Barnes, S.; Korelskiy, D.; Hedlund, J. MFI membranes for separation of carbon dioxide from synthesis gas at high pressures. J. Membr. Sci. 2015, 486, 132-137. [CrossRef]

8. Drobek, M.; Figoli, A.; Santoro, S.; Navascués, N.; Motuzas, J.; Simone, S.; Algieri, C.; Gaeta, N.; Querze, L.; Trotta, A.; et al. PVDF-MFI mixed matrix membranes as VOCs adsorbers. Micropor. Mesopor. Mater. 2015, 207, 126-133. [CrossRef]

9. Calero, S.; Gómez-Álvarez, P. On the performance of FAU and MFI zeolites for the adsorptive removal of a series of volatile organic compounds from air using molecular simulation. Phys. Chem. Chem. Phys. 2015, 17, 26451-26455. [CrossRef]

10. Ye, P.C.; Sjöberg, E.; Hedlund, J. Air separation at cryogenic temperature using MFI membranes. Microporous Mesoporous Mater. 2014, 192, 14-17. [CrossRef]

11. Wang, C.; Liu, J.Q.; Yang, J.F.; Li, J.P. A crystal seeds-assisted synthesis of microporous and mesoporous silicalite-1 and their $\mathrm{CO}_{2} / \mathrm{N}_{2} / \mathrm{CH}_{4} / \mathrm{C}_{2} \mathrm{H}_{6}$ adsorption properties. Microporous Mesoporous Mater. 2017, 242, 231-237. [CrossRef]

12. Wang, X.F.; Cui, Y.Z.; Wang, Y.; Song, X.W.; Yu, J.H. Fabrication and Catalytic Performance of Highly Stable Multifunctional Core-Shell Zeolite Composites. Inorg. Chem. 2013, 52, 10708-10710. [CrossRef] [PubMed]

13. Bare, S.R.; Kelly, S.D.; Sinkler, W.; Low, J.J.; Modica, F.S.; Valencia, S.; Corma, A.; Nemeth, L.T. Uniform Catalytic Site in Sn- $\beta$-Zeolite Determined Using X-ray Absorption Fine Structure. J. Am. Chem. Soc. 2005, 127, 12924-12932. [CrossRef] [PubMed]

14. Janda, A.; Vlaisavljevich, B.; Lin, L.C.; Smit, B.; Bell, A.T. Effects of Zeolite Structural Confinement on Adsorption Thermodynamics and Reaction Kinetics for Monomolecular Cracking and Dehydrogenation of n-Butane. J. Am. Chem. Soc. 2016, 138, 4739-4756. [CrossRef] [PubMed]

15. Singh, B.; Sinha, A.K. Synthesis of hierarchical mesoporous vanadium silicate-1 zeolite catalysts for styrene epoxidation with organic hydroperoxide. J. Mater. Chem. A 2014, 2, 1930-1939. [CrossRef]

16. O'Reilly, N.; Giri, N.; James, S.L. Porous Liquids. Chem. Eur. J. 2007, 13, 3020-3025. [CrossRef] 
17. Giri, N.; Del Pópolo, M.G.; Melaugh, G.; Greenaway, R.L.; Rätzke, K.; Koschine, T.; Pison, L.; Gomes, M.F.; Cooper, A.I.; James, S.L. Liquids with permanent porosity. Nature 2015, 527, 216-221. [CrossRef]

18. Zhang, J.; Chai, S.H.; Qiao, Z.A.; Mahurin, S.M.; Chen, J.; Fang, Y.; Wan, S.; Nelson, K.; Zhang, P.; Dai, S. Porous Liquids: A Promising Class of Media for Gas Separation. Angew. Chem. Int. Ed. 2015, 54, 932-936. [CrossRef]

19. Shan, W.; Fulvio, P.F.; Kong, L.; Schott, J.A.; Do-Thanh, C.L.; Tian, T.; Hu, X.; Mahurin, S.M.; Xing, H.; Dai, S. New Class of Type III Porous Liquids: A Promising Platform for Rational Adjustment of Gas Sorption Behavior. ACS Appl. Mater. Interfaces 2018, 10, 32-36. [CrossRef]

20. Tosheva, L.; Valtchev, V.P. Nanozeolites: Synthesis, Crystallization Mechanism, and Applications. Chem. Mater. 2005, 17, 2494-2513. [CrossRef]

21. Yan, W.F.; Chen, B.; Mahurin, S.M.; Hagaman, E.W.; Dai, S.; Overbury, S.H. Surface Sol-Gel Modification of Mesoporous Silica Materials with TiO2 for the Assembly of Ultrasmall Gold Nanoparticles. J. Phys. Chem. B 2004, 108, 2793-2796. [CrossRef]

22. Hu, X.X.; Koyanagi, T.; Fukuda, M.; Katoh, Y.; Wirth, B.D.; Snead, L.L. Defect evolution in single crystalline tungsten following low temperature and low dose neutron irradiation. J. Nucl. Mater. 2016, 470, 278-289. [CrossRef]

23. Tao, S.J. Positronium annihilation in molecular substances. J. Chem. Phys. 1972, 56, 5499-5510. [CrossRef]

24. Eldrup, M.; Lightbody, D.; Sherwood, J.N. The temperature dependence of positron lifetimes in solid pivalic acid. Chem. Phys. 1981, 63, 51-58. [CrossRef]

(C) 2019 by the authors. Licensee MDPI, Basel, Switzerland. This article is an open access article distributed under the terms and conditions of the Creative Commons Attribution (CC BY) license (http://creativecommons.org/licenses/by/4.0/). 